\title{
Analysis Of Eddy Current Loss Of IPMSM According To The Material Of Permanent Magnet
}

\author{
Byeong-Chul Lee, In-Soo song and Ki-Chan Kim* \\ Department of Electrical Engineering, Hanbat National University, Daejeon, 34158, Korea. \\ *Corresponding author.; Email address: kckim@hanbat.ac.kr
}

\begin{abstract}
Article History:Received:11 november 2020; Accepted: 27 December 2020; Published online: 05 April 2021
Abstract : In order to reach the performance of the permanent magnet embedded rotor, the choice of magnet is very important. It must be thermally stabilized, and at this point, discussion of eddy current losses is necessary. To proceed with this study, a permanent magnet embedded synchronous motor used in the compressor currently being designed was selected. To derive the eddy current losses in the neodymium-magnets, current density was calculated through the equation. The eddy current loss was mathematically derived using the magnetic conductivity and residual magnetic flux density. Finally, comparative verification was performed through finite element analysis simulation. In this paper, eddy current losses in a $\mathrm{N}$ series magnet are mathematically analyzed and we perform comparative verification through simulation using finite element analysis. The Br value indicating the residual magnetic flux density is the lowest in N30 series and the largestin the N48 series. In the case of using the N30 series, the amount of magnetic flux that can be generated is low, so in order to increase the same output, the electric field must be increased by drawing more current from the stator winding. That is, the torque can be further increased. However, since the magnetic flux density experienced by the permanent magnet also increases, eddy current loss that may occur in the magnet eventually increases. There are also a method of using a split magnet to reduce eddy current losses. Inthe case of a permanent magnet holding a large residual magnetic flux density, the magnets loss is reduced, but there is a disadvantage that the price may be expensive. The losses in the permanent magnet are dissipated as heat. If the eddy current loss increases, the magnet demagnetizes, which in turn leads to a decrease in performance. In the selection of magnets, analysis of losses is essential.
\end{abstract}

Keywords: Eddy current loss, Permanent Magnets, Magnetic flux density, Current density, IPMSM.

\section{Introduction}

IPMSM (Interior Permanent Magnet Synchronous Motor) is a synchronous motor in which the magnetic flux of a rotor is generated by a permanent magnet, which has the advantage of high efficiency and power density because there is almost no rotor loss. In addition, in recent years, with the development of control technology and the emergence, development, and mass production of rare earth magnets such as $\mathrm{NdFeB}$ holding a large residual magnetic flux density, interior $\mathrm{N}$ series magnet type synchronous motor has been frequently used[1]. Among them, IPMSM with permanent magnets embedded in the rotor is considered. Since the magnet is embedded in the rotor, it has a strong advantage even at high speed, but there is a problem of Demagnetization phenomenon for the magnet. High power density has been achieved, but this means that the loss density increases[2-5]. In other words, to minimize this loss, the loss inside the rotor must be considered. In general, the rotor loss refers to the loss of eddy currents in the iron core and the areas having conductivity. Permanent magnets have conductivity. The core loss of the rotor core is negligible. However, eddy-current loss of the $\mathrm{N}$ series magnets greatly affects motor's performance [6].

\section{Compressor motor design and analysis of losses}

2.1 Modeling a compressor motor for analysis

In this study, to analyze eddy-current loss of magnet, an IPMSM compressor with a power of $4.628 \mathrm{~kW}$ was selected as the basic model. The detailed specifications are shown in Table 1. For analysis, the model was modeled in $2 \mathrm{~d}$ and $3 \mathrm{~d}$ as shown in Fig. 1, and the shape of the magnet embedded in the rotor is called a delta type. In order to increase the magnetic flux density efficiently, a bar type is inserted on the outside, and a V type is inserted below. The main losses of permanent magnet synchronous motors are largely divided into copper loss and core loss. This losses are mainly generated as heat, and adversely affects the performance of the electric motor[7]. In this paper, we analyze the temperature that changes depending on the type of neodymium magnet.

*Corresponding author: Ki-Chan Kim

Department of Electrical Engineering, Hanbat National University, Daejeon, 34158, Korea.

Email address: kckim@hanbat.ac.kr 

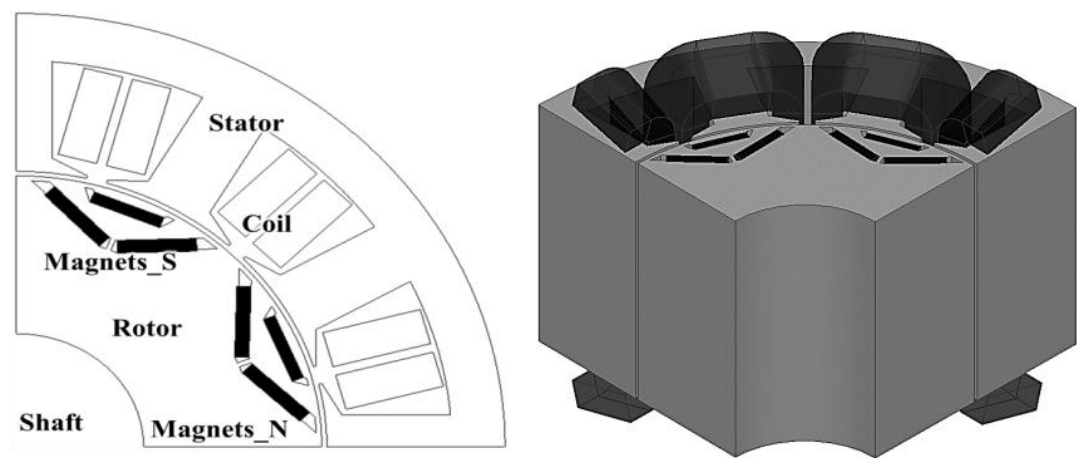

Figure 1 2d, 3d modeling of the compressor motor for electromagnetic field analysis and characterization

Table1 IPMSM Specifications

\begin{tabular}{|l|l|l|}
\hline Parameter & Value & Unit \\
\hline Motor type & IPMSM & - \\
\hline Pole/slot & $8 / 12$ & - \\
\hline Stator dia. / Rotor dia. & $96 / 60$ & $\mathrm{~mm}$ \\
\hline Speed & $\begin{array}{l}\text { Rated speed: } 6500 \\
\text { Max speed : 9000 }\end{array}$ & $\mathrm{rpm}$ \\
\hline Magnet & NdFeB(N45SH) & - \\
\hline
\end{tabular}

\subsection{Compressor motor loss analysis}

\subsubsection{Copper loss}

When the motor is running, the loss caused by current being applied to the coil in the stator is called copper loss. The copper loss in winding can be expressed by the (1) formula. The reason for multiplying 3 in Equation (1) is that the current and resistance here mean phase current and phase resistance, respectively. As shown in equation (2), $\rho$ is the resistivity of the conductor, and lengths $S$ and 1 represent cross-sectional area of the conductor and the length respectively. As can be seen from equations (1) and (2), P(copper loss) consists of the product of the square of the phase current and the resistance. Resistance is proportional to the conductivity and length of the conductor, and inversely proportional to the cross-sectional area of the winding.

$$
P_{\text {copper loss }}=3 I^{2} R
$$

\subsubsection{Figure and tables}

$$
R=\rho \frac{l}{S}
$$

Core losses are a loss caused by a change in the direction of magnetization in an core, and is generally expressed as Equation (3). Each Bm represents the max-value of magnetic flux density, and ke represents eddy current loss coefficient, $\mathrm{kh}$ is the hysteresis loss coefficient, and ka represents the drift load loss coefficient. As you can see from this formula, there is no term for current. In other words, core loss means no-load loss that always exists regardless of current. Since the drift load loss is very small, it is neglected in this paper.

$$
P_{\text {core loss }}=k_{h} f B_{m}^{2}+k_{e} f^{2} B_{m}^{2}+k_{a} f^{1.5} B_{m}^{1.5}
$$

\subsubsection{Equation of Eddy current loss}

Eddy current loss, when a magnetic body is magnetized by an external magnetic field, current is generated in a direction to prevent magnetization inside the magnetic body, which is defined as eddy current. In IPMSM, eddy current loss occurs mainly in materials with conductivity. Excessive loss is expressed as heat, which causes problems such as demagnetization and reduced motor efficiency. That is, it is essential to sufficiently consider eddy current loss at the design stage. The eddy current loss can be expressed as Equation (4) and Equation (5) is the term for the eddy current loss coefficient. Here, each $t$ is the length of the stack, $\rho$ is the resistivity, and D is the density. The unit of eddy current loss is loss per $\mathrm{kg}$. It can be seen that, as shown in Equations (4) and (5), if the resistivity is high, the stacking length of the iron core is reduced, the frequency is reduced, or the magnetic flux density is lowered, the loss of eddy currents can be effectively reduced. For the accurate thermal analysis of this paper, we will pay attention to this eddy current loss.

$$
\begin{aligned}
P_{e} & =k_{e} f^{2} B_{m}^{2} \\
k_{e} & =\frac{\pi^{2} t^{2}}{6 \rho D}
\end{aligned}
$$




\subsection{Interpretation of eddy current loss according to magnet-material}

2.3.1 Basic waveform on neodymium magnet $\mathrm{N45SH}$

Table 2 represents the permanent magnet specifications of the compressor motor that is basically applied. The thermal coefficient of $\mathrm{Br}$ is -0.12 , and the residual magnetic flux density decreases at a rate of $0.12 \%$ for every 1 degree Celsius increase. In this paper, since it is calculated based on 100 degrees Celsius, the value calculated by Equation (6) is applied.

Table2 N45SH Specification

\begin{tabular}{|l|l|l|}
\hline & Value & Unit \\
\hline Br, Residual Flux Density & 1.35 & $\mathrm{~T}$ \\
\hline HcB, Coercivity & 12750 & Oe \\
\hline Electrical Resistivity & 180 & $\mu \Omega * \mathrm{~cm}$ \\
\hline Recoil permeability & 1.04 & - \\
\hline Specific Heat & 460 & $\mathrm{~J} /(\mathrm{Kg} * \mathrm{~K})$ \\
\hline $\begin{array}{l}\mathrm{TC}, \text { Temperature Coefficients of } \\
\mathrm{Br}\end{array}$ & -0.12 & $\% / \mathrm{C}$ \\
\hline
\end{tabular}

$$
B_{r, 100}=B_{r}\left(1-\left(10^{-2} T C\right) *(100-20)\right)
$$

Analyze a basic compressor motor with magnet N45SH applied. Fig. 2(a) shows the T-N curve of the compressor motor. $6.8 \mathrm{Nm}$ is required at $6500 \mathrm{rpm}$, and at this time, an output of 4.628 is generated. After that, it enters the constant output area, and the maximum speed is $9000 \mathrm{rpm}$. Fig. 2(b) shows the torque waveform at the rated speed and maximum speed, respectively. As a result of FEM analysis, a torque of $6.82 \mathrm{Nm}$ is generated at the rated speed, and the torque ripple is quite small.

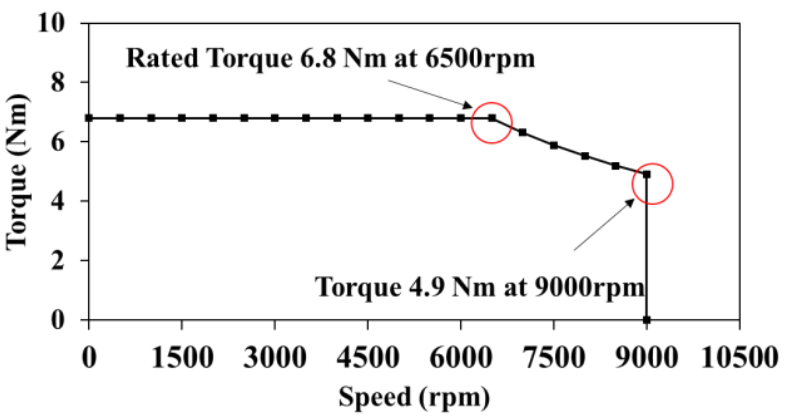

(a)

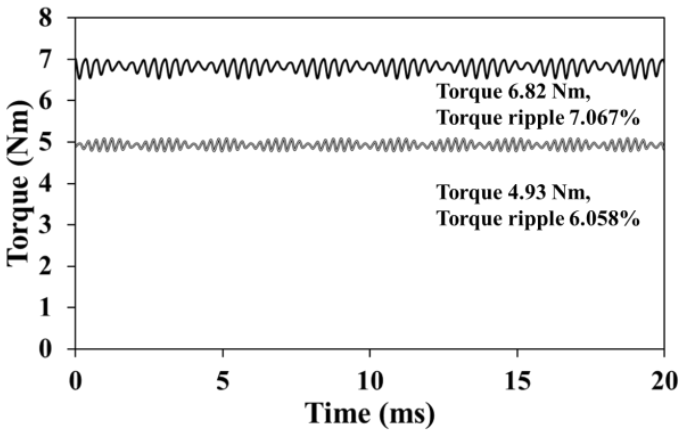

(b)

Figure 2 (a) Required curve for Compressor motor. (b) Torque Waveform for Compressor motor.

\subsubsection{PM's eddy current loss}

The faster a rotor rotates, the greater losses of eddy current in the $\mathrm{N}$ series permanent magnet inserted at the rotor. This is because the faster the speed, the faster the magnetic flux linking to the magnet with conductivity changes, and the inside of the magnet interferes with this. Induction current is generated, which is called eddy current. This current causes a loss in the magnet, which can be expressed by an equation. The vector magnetic potential can be used in all regions where the current density is zero or non-zero, and can be used in a field having a temporal change[8-13]. The vector magnetic potential is given by Equation (7). Since the divergence for rotation of all vector systems is always 0, considering Equation (7), the magnetic flux density can be expressed as Equation (8).

$$
\begin{aligned}
& \nabla \cdot \hat{B}=0 \\
& \hat{B}=\nabla \times \hat{A}
\end{aligned}
$$

$\mathrm{A}$ is the vector magnetic potential. $\mathrm{B}$ is the magnetic flux density. Meanwhile, Ampere's law can be expressed by Equation (9). If modified by the formula of the current density, it is as follows.

$$
\begin{array}{r}
I=\frac{1}{\mu} \oint \nabla \times \hat{A} \cdot d \hat{L} \\
J=\frac{1}{\mu S} \oint \nabla \times \hat{A} \cdot d \hat{L}
\end{array}
$$

Eddy current loss can be calculated by Equation (11). Here, $\sigma$ means the conductivity of the magnet. Fig. 3 shows the current density of each magnets when magnet $\mathrm{N} 45 \mathrm{SH}$ is used. Fig. 3 shows the distribution of three magnets inserted in one pole. By equation (10), the current densities of magnets A (the top bar shape), B (the bar shape on the left side), and $\mathrm{C}$ (the bar shape on the right side) can be calculated by equation (10). It can be seen 
that magnet $\mathrm{C}$ has a relatively high current density, which means that magnetic flux density is intensively distributed in area as it rotates. Eddy current losses are also expected to be large.

$$
P_{E d d y}=\frac{1}{\sigma} \int J^{2} d v
$$

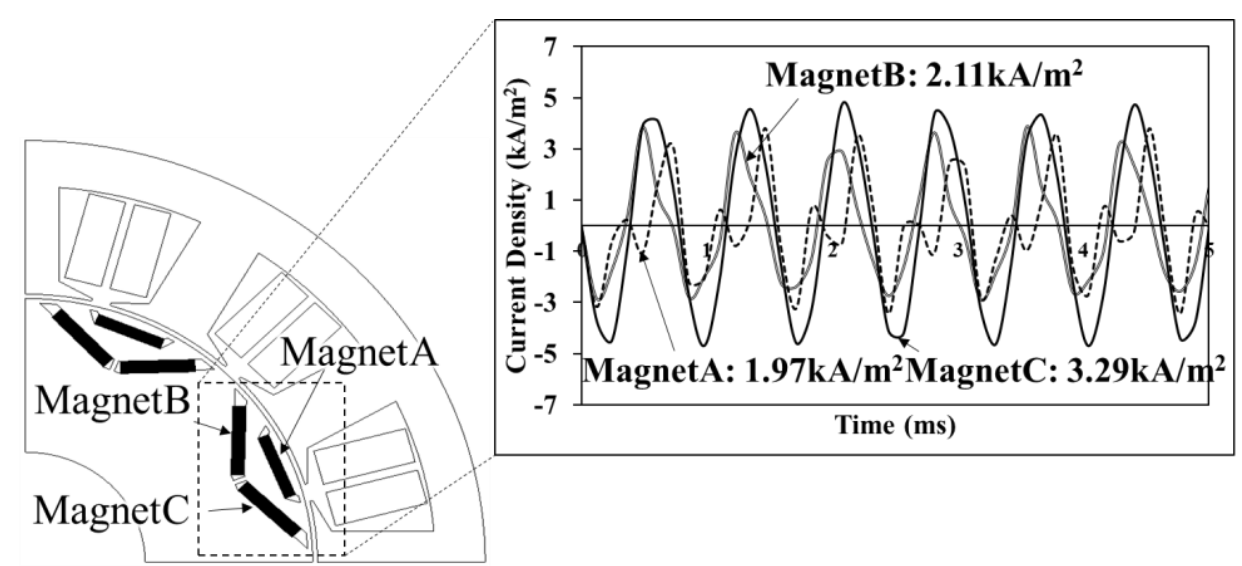

Figure 3 Current density distribution for each of the three magnets in one pole
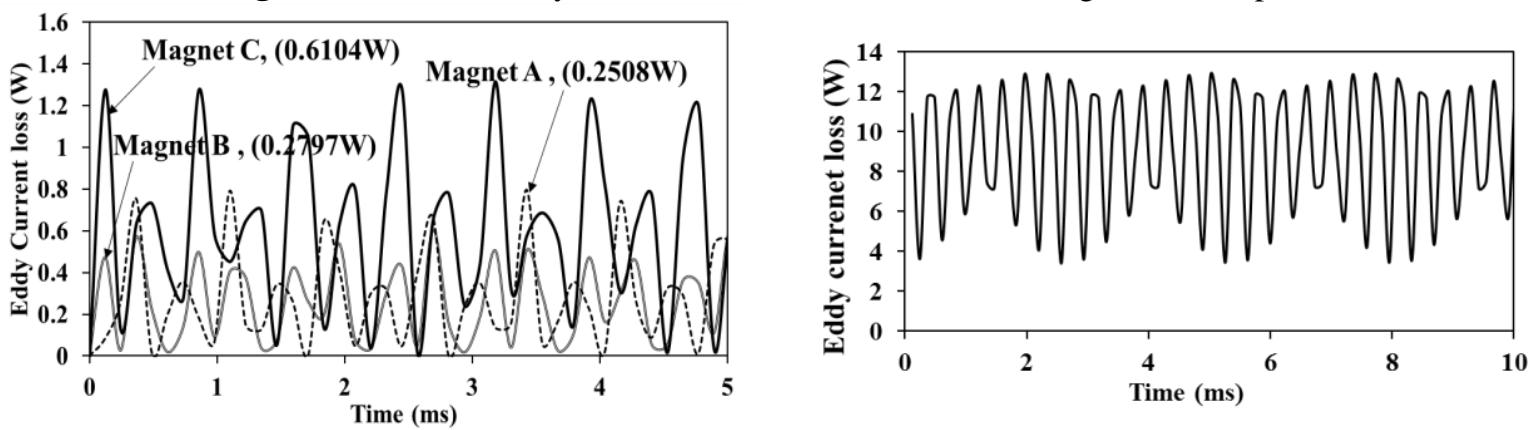

Figure 4 Eddy current loss distribution for each of the three magnets in one pole and Total eddy current loss distribution at eight poles

Fig. 4 represents eddy-current loss into each magnet $\mathrm{A}, \mathrm{B}, \mathrm{C}$ at one pole. It can be calculated by Equation (11), and the average loss of the magnet $\mathrm{C}$ (in the form of a bar on the right side) is $0.6104 \mathrm{~W}$, which has a high eddy current loss because magnetic flux generating torque are crowded and the magnetic flux density is relatively large. When the average eddy current loss of one pole is calculated, it is $1.1409 \mathrm{~W}$, and if it is multiplied by 8 because it is a total of eight poles, the losses of 9.1272 is calculated as shown in Fig. 5.

Table3 Specification of the Neodymium magnets for simulation

\begin{tabular}{|c|c|c|c|c|}
\hline Parameter & N30SH & N45SH & N48SH & Unit \\
\hline Br(at 20C) & 1.125 & 1.35 & 1.39 & $\mathrm{~T}$ \\
\hline HcB, Coercivity(at 20C) & 10700 & 12750 & 13050 & Oe \\
\hline Electrical Resistivity & 180 & 180 & 180 & $\mu \Omega^{*} \mathrm{~cm}$ \\
\hline Recoil permeability & 1.04 & 1.04 & 1.04 & - \\
\hline Specific Heat & 460 & 460 & 460 & $\mathrm{~J} /(\mathrm{Kg} * \mathrm{~K})$ \\
\hline $\begin{array}{c}\text { Reversible } \\
\text { Temperature Coefficients }\end{array}$ & -0.12 & -0.12 & -0.12 & $\% / \mathrm{C}$ \\
\hline
\end{tabular}

Table 3 shows the specifications of magnets to investigate eddy current loss by material. B_r value representing the residual magnetic flux density is the smallest in N30SH. If a magnet made of N30SH material with the lowest residual magnetic flux density are used, the amount of magnetic flux that can be generated by the magnet is low, so more current must be applied. In order to maintain the same output when the conventional N45SH magnet is used, more current is injected from the winding of the faulty member, which increases the stator magnetism. The losses in the magnet is proportional to the square of frequency and maximum magnetic flux density as shown in Equations (4),(5). Although the frequency and eddy current loss coefficient are the same, the maximum magnetic flux density is the largest in $\mathrm{N} 30 \mathrm{SH}$ as shown in Fig. 5 because the magnetomagnetic force generated by the stator is large. In other words, the magnet experiences a greater maximum magnetic flux density. This phenomenon causes an increase in the eddy current loss of the magnet. 


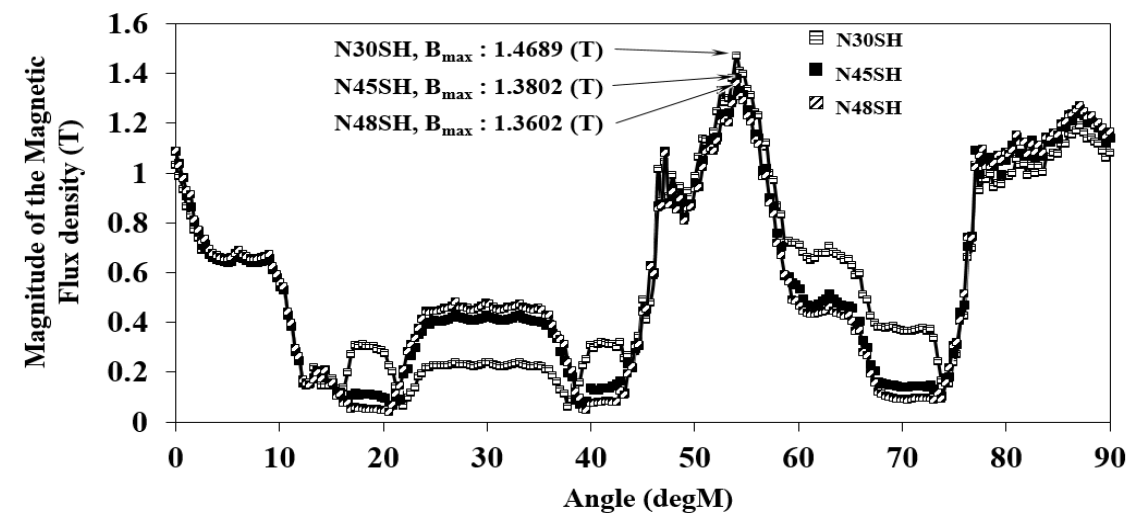

Figure 5 Graph of magnetic flux density for each magnet according to the machine angle

Fig. 6 represents sum of eddy current losses according to $\mathrm{N}$ series magnet material. Eddy current loss is lowest when N48 series magnets with a large residual magnetic flux density is used. It can sufficiently generate the required torque, which does not require a high current. In other words, the magnetic flux density experienced by the magnet is low. Fig. 7 represents the distribution of losses according to the materials of N30SH, N45SH, and $\mathrm{N} 48 \mathrm{SH}$. When N48SH magnet is used, it can be seen that it generates the least eddy current loss. The reason for the small loss is that the residual magnetic flux density is the largest, so that a large amount of current on the stator side is not required to generate the same output. That is, since a large amount of magnetic force on the stator side is not required, the maximum magnitude of the external magnetic flux density experienced by the magnet is the lowest. When N30SH magnet is used, the most eddy current loss occurs. Contrary to what was mentioned previously, more current is applied because more current is required to generate the same output. This phenomenon eventually increases the maximum magnetic flux density experienced by the magnet and causes a large eddy current loss.

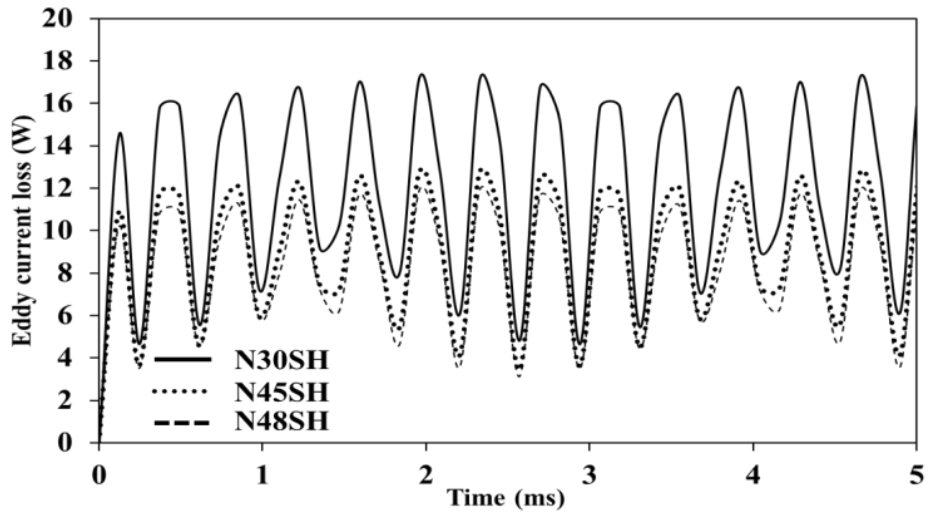

Figure 6 Total sum of each eddy current loss at 8 poles according to magnet material

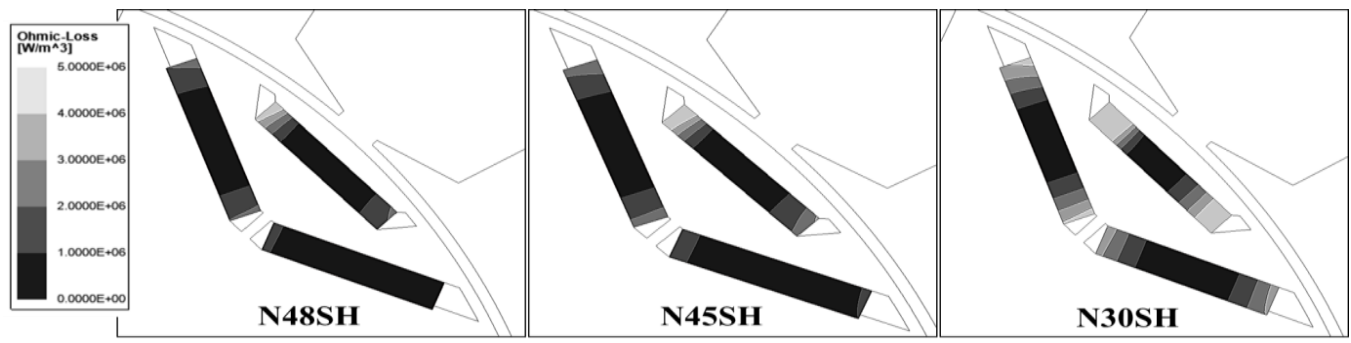

Figure 7 Eddy current losses distribution according to $\mathrm{N}$ Series permanent magnets material

\section{Conclusion}

In this study, eddy current loss was analyzed by applying magnets having different residual magnetic flux densities using the equation of eddy current loss. With the discovery of neodymium-based magnets with high residual magnetic flux density, high power density has been achieved by utilizing a type of motor embedded with the PM. As the characteristics of the magnet become important, there is a need to analyze the eddy current loss generated by magnets. If a magnet with a low residual magnetic flux density is used, the unit cost will be reduced, but a situation arises in which stator current must be input as much in order to exhibit the same 
characteristics. This causes an increase in the magnetic force on the stator side, and eventually, the magnetic flux density experienced by the permanent magnet increases, and thus the eddy current loss increases. The occurrence of eddy current loss is eventually released as heat, which will adversely affect the motor performance, so it is necessary to properly select a permanent magnet.

\section{References}

1. Yo Sakaki, S. Imagi. Relationship among eddy current loss, frequency, maximum flux density and a new parameter concerning the number of domain walls in polycrystalline and amorphous soft magnetic materials. IEEE Trans. Magn. 1981;17:1478-1480.

2. Y. Hu, S. Zhu, and C. Liu. Magnet eddy-current loss analysis of interior PM machines for electric vehicle application. IEEE Trans. Magn. 2017;3:7402904.

3. M. T. Kakhki, J. Cros, and P. Viarouge. New approach for accurate prediction of eddy current losses in laminated material in the presence of skin effect with 2-D FEA. IEEE Trans. Magn. 2016;52:6300604.

4. S. Xue, J. Feng, S. Guo, J. Peng, W. Q. Chu, and Z. Q. Zhu. A new iron loss model for temperature dependencies of hysteresis and eddy current losses in electrical machines. IEEE Trans. Magn. 2018;54:8100310.

5. Y. Aoyama, K. Miyata, and K. Ohashi. Simulations and experiments on eddy current in Nd-Fe-B magnet. IEEE Trans. Magn. 2005;41:3790-3792.

6. K. Guo. Irreversible demagnetization analysis of permanent magnet materials in a novel flux reversal linear-rotary permanent magnet actuator. IEEE Trans. Magn. 2016;52:8202904.

7. Y. Kawase, T. Ota, and H. Fukunaga. 3-D eddy current analysis in permanent magnet of interior permanent magnet motors. IEEE Trans. Magn. 2000;36:1863-1866.

8. T. Lubin, S. Mezani, and A. Rezzoug. 2-D exact analytical model for surface-mounted permanent-magnet motors with semi-closed slots. IEEE Trans. Magn. 2011;47:479-492.

9. Kameari. Calculation of transient 3-D eddy current using edgeelements. IEEE Trans. Magn. 1990;26:466469.

10. T. Kabashima, A. Kawahara, and T. Goto. Force calculation using magnetizing currents. IEEE Trans. Magn. 1988;24:451-454.

11. N. Takahashi, H. Shinagawa, D. Miyagi, Y. Doi, and K. Miyata. Analysis of eddy current losses of segmented Nd-Fe-B sintered magnets considering contact resistance. IEEE Trans. Magn. 2009;45:12341237.

12. Bisoy, S. K., Mallick, P. K., \& Mishra, A. Fairness Analysis of TCP Variants in Asymmetric Network. International Journal of Engineering \& Technology, 7(2.12), 231-233.

13. Mishra, S., Mallick, P. K., Tripathy, H. K., Bhoi, A. K., \& González-Briones, A. (2020). Performance Evaluation of a Proposed Machine Learning Model for Chronic Disease Datasets Using an Integrated Attribute Evaluator and an Improved Decision Tree Classifier. Applied Sciences, 10(22), 8137. 